\title{
Energy deposit of muon bundles detected at various zenith angles in the Cherenkov water calorimeter
}

\author{
R.P. Kokoulin ${ }^{1, a}$, N.S. Barbashina ${ }^{a}$, A.G. Bogdanov ${ }^{a}$, D.V. Chernov ${ }^{a}$, \\ S.S. Khokhlov ${ }^{a}$, V.A. Khomyakov ${ }^{a}$, V.V. Kindin ${ }^{a}$, K.G. Kompaniets ${ }^{a}$, G. Mannocchi ${ }^{b}$, \\ A.A. Petrukhin ${ }^{a}$, O. Saavedra ${ }^{c}$, G. Trinchero ${ }^{b}$, V.V. Shutenko ${ }^{a}$, I.I. Yashin ${ }^{a}$, \\ E.A. Yurina ${ }^{a}$ \\ ${ }^{a}$ National Research Nuclear University MEPhI (Moscow Engineering Physics Institute), \\ 115409 Moscow, Russia \\ ${ }^{b}$ Osservatorio Astrofisico di Torino - INAF, 10025 Torino, Italy \\ ' Dipartimento di Fisica dell' Universita di Torino, 10125, Torino, Italy \\ E-mail: RPKokoulin@mephi.ru
}

\begin{abstract}
In order to solve the problem of the excess of multi-muon events observed in several cosmic ray experiments at ultra-high energies (so-called 'muon puzzle'), investigations of the energy characteristics of the muon component of extensive air showers (EAS) are required. A possible approach to such investigations is the measurement of the energy deposit of muon bundles in the detector, which provides information on the mean muon energy. In the experiment being now conducted at the NEVOD-DECOR complex, the local muon density and EAS arrival direction are determined according to the data of the coordinate-tracking detector DECOR, whereas the energy deposit is measured by means of the Cherenkov water calorimeter NEWOD. Simultaneous detection of muon bundles in a wide range of zenith angles and muon multiplicities ensures exploration of primary particle energy region of $10^{16}-10^{18} \mathrm{eV}$ and even higher. Results of the measurements of the energy deposit of the inclined muon bundles at various zenith angles based on the data accumulated during more than 29,000 h observations over the period from May 2012 to February 2017 are presented and compared with simulations performed by means of the CORSIKA program.
\end{abstract}

35th International Cosmic Ray Conference - ICRC2017

10-20 July, 2017

Bexco, Busan, Korea

\footnotetext{
${ }^{1}$ Speaker 


\section{Introduction}

Unexpectedly high rate of high-multiplicity muon bundle events was found in cosmic ray experiments conducted at the LEP detectors ALEPH [1] and DELPHI [2]. That time, it was interpreted as an indication for a heavy mass composition at very high energies of primary particles. However, the analysis of DECOR data $[3,4]$ on inclined muon bundles with a high multiplicity at large zenith angles showed that the measured event intensity cannot be explained in frame of the existing hadron interaction models even under the assumption of extremely heavy (pure iron nuclei) mass composition at primary energies around $10^{18} \mathrm{eV}$. Later, studies of the muon content in inclined extensive air showers (EAS) in the energy range of $10^{19} \mathrm{eV}$ in the Pierre Auger Observatory [5, 6] confirmed the excessive amount of muons: considerably more muons in simulations were needed to match the data and simulations. Now, the inconsistence muon abundance in EAS at ultra-high energies is often referred to as "muon problem" or "muon puzzle".

It is naturally to assume that for explanation of the observed excess of multi-muon events the existing models of the development of the nuclear cascade in the atmosphere need a revision. A key to the solution of this problem may give investigations of the energy characteristics of the muon component of EAS and their variations with the primary energy [7]. A possible approach to such investigations is the measurement of the energy deposit of muon bundles in the detector material. Specific energy loss of muons in the region above hundreds $\mathrm{GeV}$ almost linearly increases with muon energy, and the appearance of excessive flux of high-energy muons should change the dependence of the average muon bundle energy deposit on the energy of the primary particle. An experiment on investigations of the energy deposit of inclined muon bundles was started at the Experimental complex NEVOD in 2012. The complex includes the Cherenkov water calorimeter $[8,9]$ and the coordinate-tracking detector [10] which ensures a reliable identification of multi-muon events. Detection of muon bundles in a wide range of multiplicities and zenith angles provides a possibility of exploration of a very wide interval of primary energies in frames of a single experiment. Preliminary results of the measurements of muon bundle energy deposit were reported at the preceding conference [11]. For the moment, the available experimental statistics has been increased about 1.7 times and is now based on 29,175 $h$ of net observation time. In the paper, updated results of the NEVOD-DECOR experiment on muon bundle energy deposit measurements are presented and compared with simulations performed on the basis of the CORSIKA code [12].

\section{Experimental data}

The Cherenkov water detector (CWD) NEVOD [8] has an inner volume of $9 \times 9 \times 26$ cubic meters and is equipped with a spatial lattice of quasi-spherical measuring modules (QSM). Each QSM includes six FEU-200 photomultipliers with flat $15 \mathrm{~cm}$ diameter cathodes directed along the axes of the orthogonal coordinate system. In total, the measuring system contains 91 QSMs (546 PMTs), arranged in vertical strings ( 3 or 4 QSMs in each) with distances $2.5 \mathrm{~m}$ along the main axis of the water tank and $2.0 \mathrm{~m}$ across it and over the depth. After a recent modernization [9], electronic systems of the detector ensure measurements of the signals from each PMT in a wide dynamic range from 1 to $10^{5}$ photoelectrons. The setup includes also calibration telescope system (CTS); 40 plastic scintillation counters with area $20 \times 40 \mathrm{~cm}^{2}$ are located on the roof of 
the water tank, above the QSM lattice, and 40 counters on the tank bottom. Coordinate-tracking detector DECOR [10] with a total area about $70 \mathrm{~m}^{2}$ includes eight supermodules (SMs) deployed in the galleries of the NEVOD building from three sides of the water tank. The sensitive area of each supermodule is $8.4 \mathrm{~m}^{2}$. Supermodules consist of 8 vertical planes of plastic streamer tube chambers with resistive cathode coating and two-dimensional system of external readout strips. The spacing between neighboring planes is $6 \mathrm{~cm}$. The spatial and angular accuracy of muon track reconstruction in the SM is better than $1 \mathrm{~cm}$ and $1^{\circ}$, respectively. A general layout of the setup with an example of the muon bundle event detection is shown in Figure 1.

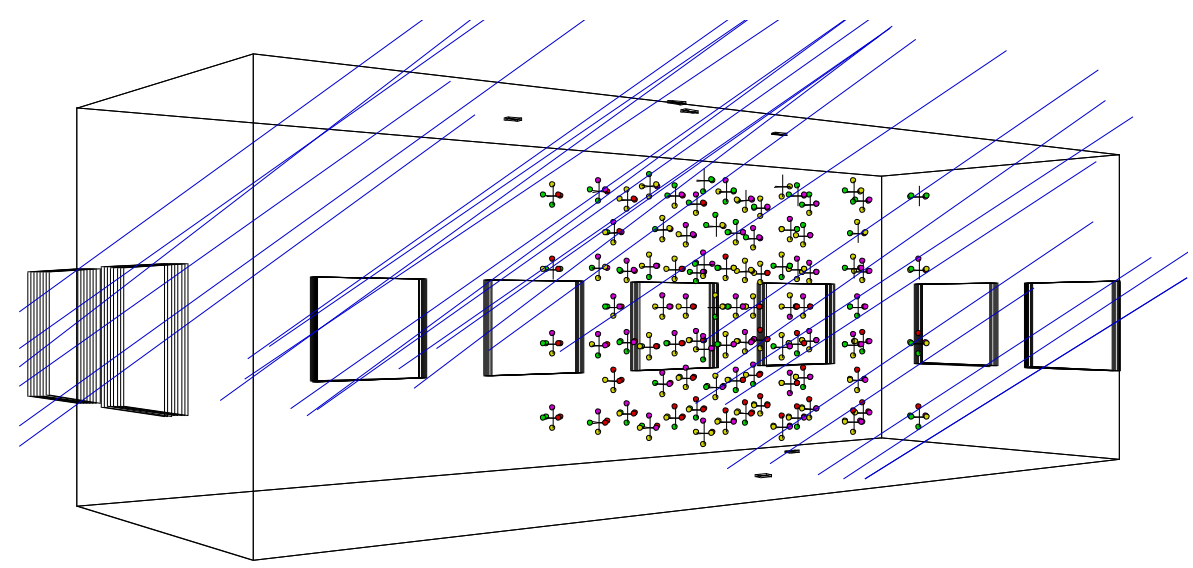

Figure 1: An example of the muon bundle event detected in the NEVOD-DECOR complex. Thin lines represent muon tracks reconstructed from DECOR data; small circles indicate hit photomultipliers of the CWD; big rectangles around the CWD volume are DECOR SMs; small dark rectangles at the top and the bottom represent hit counters of the CTS.

In the present analysis, data of three measurement series conducted during a nearly five-year long period between May 2012 and February 2017 are used (measurements are now being continued). The total live time of observations amounted to $29,175 \mathrm{~h}$. In these data, 49460 muon bundle events with muon multiplicities $m \geq 5$ and zenith angles $\theta \geq 55^{\circ}$ have been found. In addition, from the initial part of the accumulated experimental material ( $3253 \mathrm{~h} \mathrm{live} \mathrm{time),} \mathrm{muon}$ bundles arriving at moderate zenith angles were selected: 15084 events with $m \geq 5$ and $40^{\circ} \leq \theta<55^{\circ}$. The events were selected in two $60^{\circ}$-wide sectors of the azimuth angle where most of DECOR supermodules (six of eight) were screened with the NEVOD water volume; data of only these shielded SMs were used for muon track counting. The average muon threshold energy for such selection conditions is about $2 \mathrm{GeV}$.

As a measure of the muon bundle energy deposit in the NEVOD, the sum $\Sigma$ of the signals of all hit PMTs of the water calorimeter is used (in units of photoelectrons, ph.e.). It is assumed that the total yield of Cherenkov photons is proportional to the total energy loss of muons in the detector material (including the energies of secondary particles produced by muons and cascades from them). The local density of muons $D\left(\mathrm{~m}^{-2}\right)$ in the event is estimated on the basis of the number of the detected muons and effective area $S_{\text {det }}$ of six DECOR SMs for the measured direction of muon bundle arrival. A preliminary analysis showed that, as it might be expected, in a first approximation the total energy deposit in CWD is proportional to the local muon density. Therefore, further we consider the specific energy deposit $\Sigma / D$, i.e. the Cherenkov calorimeter response normalized to the local muon density estimate in the event. 


\section{Results and discussion}

Results of the measurement of zenith-angular dependence of the average specific energy deposit of muon bundles are presented by the points in Figure 2. At moderate zenith angles (less than $55^{\circ}$ ), a residual contribution of electromagnetic and hadron EAS components to the response of the unscreened water calorimeter located at the surface is observed. This contribution decreases nearly exponentially with the slant depth of the atmosphere [11], and at large zenith angles practically only muons and secondary relative to them particles remain in the bundle events. Arrows in the lower part of the figure indicate typical energies (calculated meanlogarithmic values [4]) of primary particles contributing to muon bundle formation at different zenith angles. As seen from the figure, experimental data clearly demonstrate the increase of the specific energy deposit with zenith angle.

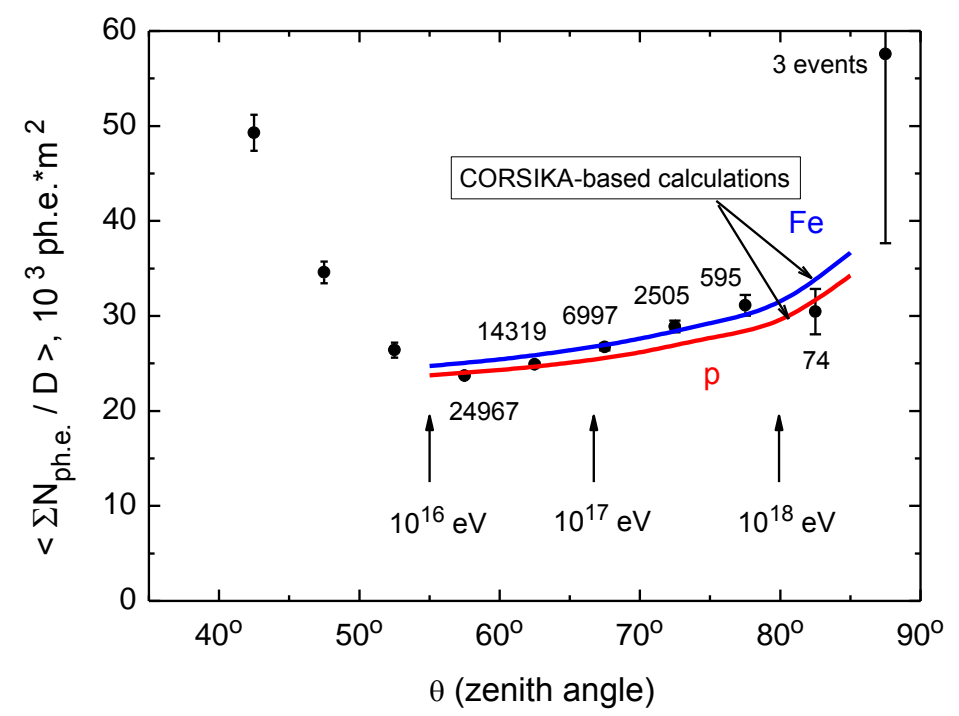

Figure 2: Dependence of the average specific energy deposit of muon bundles on the zenith angle. The points correspond to the present data; the numbers of events in respective bins are indicated. The curves represent results of CORSIKA-based calculations for primary protons and iron nuclei (labels 'p' and 'Fe', respectively).

The curves in Figure 2 represent the expected angular dependence of the specific energy deposit obtained on the basis of simulation of EAS muon component by means of the CORSIKA code [12] for protons and iron nuclei as primary particles. In simulations, we have used the combination of hadron interaction models SIBYLL2.3 [13] and FLUKA [14] for hadrons with energies above and below $80 \mathrm{GeV}$, respectively. As calculations show, the value of the mean energy of muons comprising the bundles rapidly increases with zenith angle and reaches approximately $500 \mathrm{GeV}$ at $\theta=85^{\circ}$. The calculated dependence of the average specific energy deposit was obtained in the following way. First, for every zenith angle the average specific muon energy loss $\langle d E / d X\rangle$ was calculated using the energy loss tables [15]. Then, the aforementioned assumption that the total Cherenkov light yield in the calorimeter is proportional to the muon energy loss was done. Finally, the absolute calibration was derived from the normalization of the calculated curve for primary protons to measurement results in the zenith angle interval $55^{\circ}-65^{\circ}$. As a whole, the measured dependence of the average specific energy deposit on the zenith angle confirms the increase of the mean muon energy in the bundles and is in a reasonable agreement with expectation. However, at angles $\theta>70^{\circ}$ (at 
effective primary energies more than about $10^{17} \mathrm{eV}$ ) some indication for an excess compared to calculations is seen, even for heavy primary nuclei assumption.

In Figure 3, the measured average specific energy deposit of muon bundles detected in zenith angle interval $55^{\circ} \leq \theta<65^{\circ}$ is plotted as a function of the local muon density. In fact, such consideration for a fixed interval of zenith angles allows to follow possible changes of the detector response (and correspondingly, of the mean muon energy in the bundles) with the increase of the primary energy. The curves in the figure are obtained on the basis of simulation for primary protons and iron nuclei for a fixed zenith angle $\theta=59^{\circ}$; the same normalization coefficient as in Figure 2 has been applied. Simulation results demonstrate a trend to a slow decrease of the mean muon energy for increasing primary energy; on the contrary, the data give a hint (though not statistically significant yet) for an increase of the specific energy deposit at primary energies exceeding $10^{17} \mathrm{eV}$.

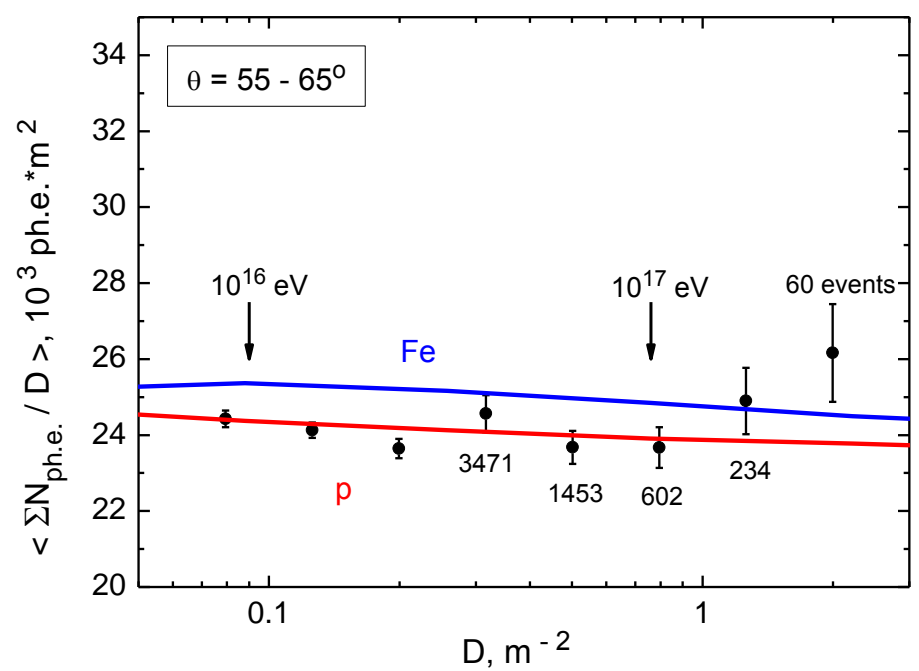

Figure 3: Dependence of the average specific energy deposit for muon bundles with arrival zenith angles $55^{\circ} \leq \theta<65^{\circ}$ on the local muon density. Notations are the same as in Figure 2.

Of course, for a more definite conclusion about an excessive amount of high-energy muons in the bundles an increase of experimental statistics and careful analysis of possible systematic distortions are required. One of the possible systematic effects may be an underestimation of the number of muons in the bundles at high muon densities because of the track masking due to a final detector resolution. This difficulty may be overcome if we analyze the sample of events corresponding to larger zenith angles. For example, if we shift the minimal zenith angle from $55^{\circ}$ to $65^{\circ}$, then approximately 3 times lower muon densities will correspond to the same primary energies, and the loss of the tracks at effective energies around $10^{17} \mathrm{eV}$ will be negligible. Simultaneously, a residual effect of non-muon components on the CWD response, if exists, will be practically eliminated due to a significant increase of the slant depth of the atmosphere. In Figure 4, the same dependence as in Figure 3 but for zenith angle interval $65^{\circ} \leq$ $\theta<75^{\circ}$ is presented. Though statistical errors for the moment are large, an evidence for an increase of the average specific energy deposit of muon bundles with increasing primary energy from $10^{17}$ to $10^{18} \mathrm{eV}$ is noticed. 


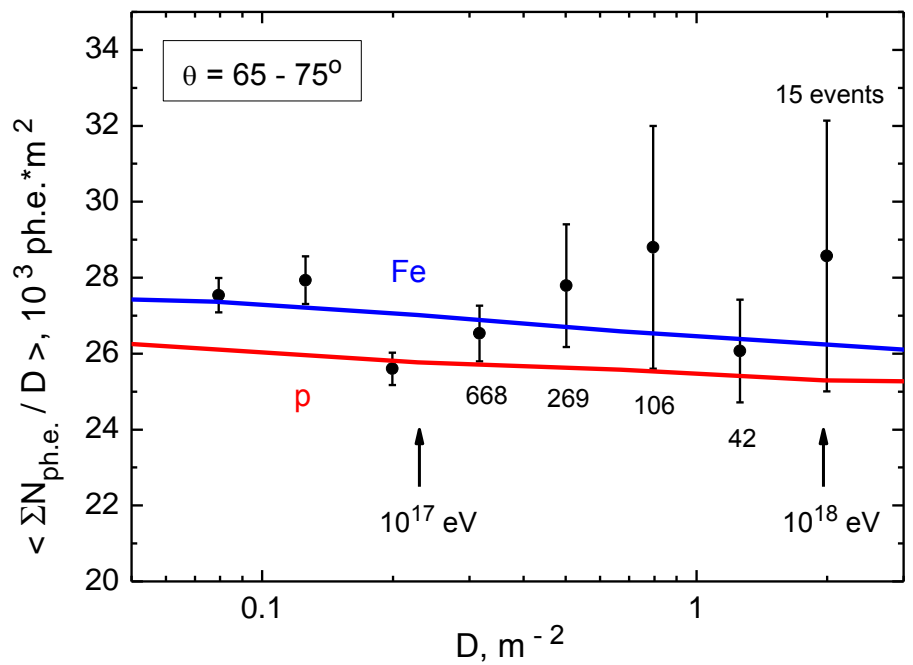

Figure 4: The same as in Figure 3, but for zenith angle interval $65^{\circ} \leq \theta<75^{\circ}$.

\section{Conclusion}

The measurements of the energy characteristics of muon bundles in inclined EAS generated by primary cosmic ray particles in the energy range $10^{16}-10^{18} \mathrm{eV}$ are being conducted at the NEVOD-DECOR complex. The preliminary results are in a reasonable agreement with CORSIKA-based simulations of EAS muon component, though some indication for an excess in the measured muon bundle energy deposit compared to calculations in the region of primary energies above $10^{17} \mathrm{eV}$ is seen. This deviation may evidence for an inclusion of a new mechanism of generation of high energy muons at ultra-high energies of primary particles.

The work is being performed at the Experimental Complex NEVOD with the support from the RF Ministry of Education and Science (contract RFMEFI59114X0002, MEPhI Academic Excellence Project, and government task).

\section{References}

[1] V. Avati et al., Astropart. Phys. 19, 513 (2003)

[2] J. Abdallah et al., Astropart. Phys. 28, 273 (2007)

[3] R.P. Kokoulin et al., Nucl. Phys. B (Proc. Suppl.) 196, 106 (2009)

[4] A.G. Bogdanov et al., Phys. Atom. Nuclei 73, 1852 (2010)

[5] L. Nellen (for Pierre Auger Collaboration), J. Phys.: Conf. Ser. 409, 012107 (2013)

[6] A. Aab et al., Phys. Rev. D 91, 032003 (2015)

[7] A.A. Petrukhin, NIM A 742, 228 (2014)

[8] V.M. Aynutdinov et al., Astrophys. Space Sci. 258, 105 (1998)

[9] S.S. Khokhlov et al., Astrophys. Space Sci. Trans. 7, 271 (2011)

[10] N.S. Barbashina et al., Instrum. Experim. Techniques 43, 743 (2000)

[11] I.I. Yashin et al., Proc. 34 $4^{\text {th }}$ Int. Cosmic Ray Conf., Hague, PoS (ICRC2015) 315 (2015)

[12] D. Heck et al., Forschungszentrum Karlsruhe Report, FZKA 6019 (1998)

[13] F. Riehn et al., Proc. $34^{\text {th }}$ Int. Cosmic Ray Conf., Hague, PoS (ICRC2015) 558 (2015)

[14] A. Ferrari et al., Report CERN-2005-10 (2005)

[15] D.E. Groom et al., Atom. Data and Nucl. Data Tables 78, 183 (2001) 